\title{
TU/e EmonOWEN

\section{Numerical and experimental investigation of structure-function scaling in turbulent Rayleigh-Bénard convection}

\section{Citation for published version (APA):}

Kunnen, R. P. J., Clercx, H. J. H., Geurts, B. J., Bokhoven, van, L. J. A., Akkermans, R. A. D., \& Verzicco, R. (2008). Numerical and experimental investigation of structure-function scaling in turbulent Rayleigh-Bénard convection. Physical Review E - Statistical, Nonlinear, and Soft Matter Physics, 77(1), 016302-1/13. [016302]. https://doi.org/10.1103/PhysRevE.77.016302

DOI:

10.1103/PhysRevE.77.016302

Document status and date:

Published: 01/01/2008

\section{Document Version:}

Publisher's PDF, also known as Version of Record (includes final page, issue and volume numbers)

\section{Please check the document version of this publication:}

- A submitted manuscript is the version of the article upon submission and before peer-review. There can be important differences between the submitted version and the official published version of record. People interested in the research are advised to contact the author for the final version of the publication, or visit the $\mathrm{DOI}$ to the publisher's website.

- The final author version and the galley proof are versions of the publication after peer review.

- The final published version features the final layout of the paper including the volume, issue and page numbers.

Link to publication

\section{General rights}

Copyright and moral rights for the publications made accessible in the public portal are retained by the authors and/or other copyright owners and it is a condition of accessing publications that users recognise and abide by the legal requirements associated with these rights.

- Users may download and print one copy of any publication from the public portal for the purpose of private study or research.

- You may not further distribute the material or use it for any profit-making activity or commercial gain

- You may freely distribute the URL identifying the publication in the public portal.

If the publication is distributed under the terms of Article 25fa of the Dutch Copyright Act, indicated by the "Taverne" license above, please follow below link for the End User Agreement:

www.tue.nl/taverne

Take down policy

If you believe that this document breaches copyright please contact us at:

openaccess@tue.nl

providing details and we will investigate your claim. 


\title{
Numerical and experimental investigation of structure-function scaling in turbulent Rayleigh-Bénard convection
}

\author{
R. P. J. Kunnen, ${ }^{1, *}$ H. J. H. Clercx, ${ }^{1,2}$ B. J. Geurts, ${ }^{1,2}$ L. J. A. van Bokhoven, ${ }^{1}$ R. A. D. Akkermans, ${ }^{1}$ and R. Verzicco ${ }^{3}$ \\ ${ }^{1}$ Fluid Dynamics Laboratory, Department of Physics, International Collaboration for Turbulence Research (ICTR) \& J. M. Burgers Center \\ for Fluid Dynamics, Eindhoven University of Technology, P.O. Box 513, 5600 MB Eindhoven, The Netherlands \\ ${ }^{2}$ Department of Applied Mathematics \& J. M. Burgers Center for Fluid Dynamics, University of Twente, P.O. Box 217, \\ 7500 AE Enschede, The Netherlands \\ ${ }^{3}$ Politecnico di Bari, DIMeG and CEMeC, Via Re David 200, 70125 Bari, Italy
}

(Received 11 October 2007; revised manuscript received 5 December 2007; published 8 January 2008)

\begin{abstract}
Direct numerical simulation and stereoscopic particle image velocimetry of turbulent convection are used to gather spatial data for the calculation of structure functions. We wish to add to the ongoing discussion in the literature whether temperature acts as an active or passive scalar in turbulent convection, with consequences for structure-function scaling. The simulation results show direct confirmation of the scalings derived by Bolgiano and Obukhov for turbulence with an active scalar for both velocity and temperature statistics. The active-scalar range shifts to larger scales when the forcing parameter (Rayleigh number) is increased. Furthermore, a close inspection of local turbulent length scales (Kolmogorov and Bolgiano lengths) confirms conjectures from earlier studies that the oft-used global averages are not suited for the interpretation of structure functions. In the experiment, a characterization of the domain-filling large-scale circulation of confined convection is carried out for comparison with other studies. The measured velocity fields are also used to calculate velocity structure functions, further confirming the Bolgiano-Obukhov scalings when interpreted with the local turbulent length scales found in the simulations. An extended self-similarity analysis shows that the relative scalings are different for the Kolmogorov and Bolgiano-Obukhov regimes.
\end{abstract}

DOI: $10.1103 /$ PhysRevE.77.016302

PACS number(s): 47.27.ek, 47.80.Cb

\section{INTRODUCTION}

Convective turbulence remains the subject of many studies, as it is very commonly found in nature as well as in industry. The problem of turbulent convection is often simplified to the classical Rayleigh-Bénard setting: a fluid layer is vertically enclosed between a heated bottom wall and a cooled top wall. The dimensionless forcing parameter, the Rayleigh number, is defined as $\mathrm{Ra} \equiv g \alpha \Delta T H^{3} /(\nu \kappa)$, with $g$ the gravitational acceleration, $\alpha$ the thermal expansion coefficient of the fluid, $\Delta T$ the temperature difference between bottom and top walls, $H$ their separation, $\nu$ the kinematic viscosity of the fluid, and $\kappa$ its thermal diffusivity. Another dimensionless parameter is the Prandtl number $\sigma \equiv \nu / \kappa$, characterizing the diffusive properties of the fluid.

The scaling properties of structure functions (SFs) and/or spectra provide insight into the small-scale dynamics of turbulent flow. The influence of temperature may be felt in the scaling of turbulence statistics when compared to homogeneous isotropic turbulence. On the one hand, when considering the scalar temperature to be passive, the well-known scalings [1] proposed by Kolmogorov in 1941 (K41) are expected for velocity, with temperature SFs as predicted [2,3] by Obukhov and Corrsin (OC). On the other hand, when temperature is an active scalar, the scalings derived by Bolgiano and Obukhov (BO) [4,5] are appropriate [6,7]. For a comprehensive overview of these scalings, we refer to Ref. [8].

Measurements of SFs and spectra in turbulence are traditionally done indirectly by recording time series of velocity

\footnotetext{
*R.P.J.Kunnen@tue.nl
}

or temperature in a single point. In the interpretation of the results Taylor's frozen-turbulence hypothesis is invoked, which requires a mean velocity, large relative to its fluctuations, sweeping across the point of measurement. In most convection settings this requirement cannot be met since the mean motion is either too weak or absent. Many of the studies using temporal statistics [9-17] find a range of scales where $\mathrm{BO}$ is the valid scaling, be it experimentally or numerically, in velocity or temperature statistics. Direct spatial measurements of the scalings are rare. Evidence for BO scaling from spatial data is found through indirect methods $[18,19]$, but also by direct measurement or calculation [20-22]. However, in another recent experimental study [23] K41 and OC scalings were found exclusively. In this paper we provide arguments for the existence of a $\mathrm{BO}$ scaling range at scales larger than those investigated in Ref. [23], with evidence from both direct numerical simulation (DNS) and experiments. This indicates that temperature is indeed an active scalar.

In DNS the focus is on the calculation of turbulent length scales governing the SF scaling: viz., the Bolgiano length $L_{B}$ and the Kolmogorov length $\eta$. We perform local calculations of these length scales, contrasting the customary globally averaged estimates that use rigorous relations for the dissipation rates that follow directly from the Navier-Stokes equations [24,25].

Velocity measurements in water have been performed using a novel technique, stereoscopic particle image velocimetry (SPIV) [26]. The main asset of SPIV, compared to regular PIV, is that it provides measurements of the normal velocity component in addition to the in-plane components in planar cross-sections of the flow domain. This technique 
allows for direct spatial measurements of velocity SFs simultaneously for the three velocity components. Here we will focus on SFs of the vertical velocity. Our measurements provide direct confirmation of the theoretically predicted $\mathrm{BO}$ scaling for buoyancy-driven turbulence. An observation of the azimuthal oscillation of the domain-filling large-scale circulation (LSC) of confined convection (see, e.g., Ref. [27]) provides validation of the experimental setup.

We will first present a summary of the theoretical scaling relations for SFs in turbulent convection (Sec. II). Then the numerical setup is discussed in Sec. III, followed by results from the DNS focusing on SFs and related length scales in Sec. IV. In Sec. V the experimental procedure is presented, along with an experimental validation based upon the azimuthal oscillation of the LSC. Then the experimental SF results are presented in Sec. VI. Finally, a summary of the results with conclusions covers Sec. VII.

\section{STRUCTURE FUNCTIONS AND SCALING}

The $p$ th-order spatial velocity SF $S_{p}^{u}$ is defined as

$$
S_{p}^{u}(\mathbf{r}) \equiv\left\langle|u(\mathbf{x}+\mathbf{r})-u(\mathbf{x})|^{p}\right\rangle,
$$

where $u$ is the first component of the velocity vector $\mathbf{u}$ $=(u, v, w), \mathbf{r}$ is the separation vector, and the angular brackets denote an ensemble average over many realizations. (In this work $z$ is the vertical direction antiparallel to gravity and $w$ is thus the vertical velocity component.) Similar definitions are used for the spatial SFs of $v$ and $w$. We can now distinguish longitudinal ( $\mathbf{r}$ in line with the velocity component $u$ ) and transverse ( $\mathbf{r}$ perpendicular to $u$ ) SFs as a function of the separation $r \equiv|\mathbf{r}|$. We remark that the dependence on the position $\mathbf{x}$ vanishes only for homogeneous turbulence; in the current work, only local homogeneity is intended.

In stably stratified turbulent convection the inertial range is partially influenced by the buoyancy force. Bolgiano and Obukhov $[4,5,8]$ derived a length scale $L_{B}$, the Bolgiano scale (defined below), above which buoyancy is dominant; in this BO regime (wave numbers $k \ll 1 / L_{B}$ ) the energy spectrum displays a scaling $k^{-11 / 5}$. At wave numbers $k \gg 1 / L_{B}$ the well-known $\mathrm{K} 41 k^{-5 / 3}$ dependence is found since this regime is inertia dominated. Later it was proven $[6,7]$ that the same scalings are also valid for the unstably stratified case-e.g., Rayleigh-Bénard convection. The corresponding scaling exponents for the $p$ th-order velocity SFs are $r^{3 p / 5}$ for BO and $r^{p / 3}$ for K41 (see, e.g., Ref. [8]).

Similarly, the $p$ th-order spatial temperature SF $R_{p}$ is defined as

$$
R_{p}(\mathbf{r}) \equiv\left\langle|T(\mathbf{x}+\mathbf{r})-T(\mathbf{x})|^{p}\right\rangle .
$$

These SFs are predicted to scale as $r^{p / 5}$ for $\mathrm{BO}$ and $r^{p / 3}$ for OC [8].

The Bolgiano length $L_{B}$ is defined as

$$
L_{B} \equiv \frac{\epsilon^{5 / 4}}{(g \alpha)^{3 / 2} N^{3 / 4}},
$$

with $\epsilon(\mathbf{x}, t) \equiv \nu|\nabla \mathbf{u}(\mathbf{x}, t)|^{2}$ the turbulent kinetic energy dissipation rate and $N(\mathbf{x}, t) \equiv \kappa|\nabla T(\mathbf{x}, t)|^{2}$ the temperature vari- ance dissipation rate. Although $\epsilon$ and $N$ (and thus also $L_{B}$ ) are local quantities, there are some rigorous relations for these quantities when concerning the entire domain $[24,25]$, providing an a priori estimate of $L_{B}$. Introducing the Nusselt number $\mathrm{Nu}$ as the total heat flux normalized by its conductive part, the following estimate is readily derived [21] (valid for $\mathrm{Nu} \gg 1)$ :

$$
\left\langle L_{B}\right\rangle \approx \frac{\mathrm{Nu}^{1 / 2}}{(\sigma \mathrm{Ra})^{1 / 4}} H .
$$

The Kolmogorov length scale $\eta \equiv \nu^{3 / 4} / \epsilon^{1 / 4}$ can by similar arguments be estimated as

$$
\langle\eta\rangle=\frac{\sigma^{1 / 2}}{(\mathrm{RaNu})^{1 / 4}} H .
$$

Later, in the discussion of DNS, an inventory of the local length scales will be given along with their dependence on Ra. There it will be shown that the local and globally averaged values are quite different in practice.

\section{NUMERICAL ARRANGEMENT}

The code used for the simulations presented here has already been extensively used for investigations into turbulent convection-e.g., in Refs. [16,28,29]. Details of the numerical procedure can be found in $[28,30]$; here, we only present an overview.

DNS concerns the flow in a cylindrical domain of diameter-to-height aspect ratio $\Gamma=D / H=1$. The bottom and top walls are kept at constant temperatures with the bottom wall at a temperature $\Delta T$ higher than the top wall. The sidewall is adiabatic. All surfaces are no-slip surfaces. The flow inside this volume is governed by the Navier-Stokes and heat equations in the Boussinesq approximation under the constraint of incompressibility:

$$
\begin{gathered}
\frac{D \mathbf{u}}{D t}=-\nabla p+T \hat{\mathbf{z}}+\left(\frac{\sigma}{\mathrm{Ra}}\right)^{1 / 2} \nabla^{2} \mathbf{u}, \\
\frac{D T}{D t}=\frac{1}{(\sigma \mathrm{Ra})^{1 / 2}} \nabla^{2} T, \\
\nabla \cdot \mathbf{u}=0 .
\end{gathered}
$$

Here $D / D t$ represents the material derivative, $p$ is the pressure, $\hat{\mathbf{z}}$ is the unit vector in the axial direction counter to gravity, and $T$ is the temperature. The equations have been made dimensionless with length $H$, temperature scale $\Delta T$ (so that $T$ ranges from 0 at the top lid to 1 at the bottom lid), and the free-fall velocity $U \equiv(g \alpha \Delta T H)^{1 / 2}$.

Equations (6a)-(6c) have been solved in cylindrical coordinates $(\rho, \theta, z)$. The discretization uses second-order accurate finite-difference approximations for the derivatives in space. This system of discrete equations is then solved with a fractional-step method, and a third-order Runge-Kutta scheme is used for the time advancement.

Statistics from the simulations that are relevant for this article include the heat flux $(\mathrm{Nu})$ and the local values of the 
TABLE I. Parameters for the numerical simulations: Rayleigh and Prandtl numbers and the number of grid points in radial $\left(N_{\rho}\right)$, azimuthal $\left(N_{\theta}\right)$, and axial $\left(N_{z}\right)$ directions.

\begin{tabular}{lcc}
\hline \hline $\mathrm{Ra}$ & $\sigma$ & $N_{\rho} \times N_{\theta} \times N_{z}$ \\
\hline $1 \times 10^{8}$ & 4 & $129 \times 257 \times 257$ \\
$2 \times 10^{8}$ & 4 & $129 \times 257 \times 257$ \\
$5 \times 10^{8}$ & 4 & $129 \times 257 \times 257$ \\
$1 \times 10^{9}$ & 4 & $129 \times 257 \times 257$ \\
$5 \times 10^{9}$ & 4 & $193 \times 385 \times 385$ \\
$1 \times 10^{10}$ & 4 & $193 \times 385 \times 385$ \\
$1 \times 10^{8}$ & 6.4 & $129 \times 257 \times 257$ \\
$2 \times 10^{8}$ & 6.4 & $129 \times 257 \times 257$ \\
$5 \times 10^{8}$ & 6.4 & $129 \times 257 \times 257$ \\
$1 \times 10^{9}$ & 6.4 & $129 \times 257 \times 257$ \\
\hline \hline
\end{tabular}

dissipations $\epsilon$ and $N$. The heat flux is calculated in two ways: (i) the average wall-normal derivative of temperature, in these units $\mathrm{Nu}=\langle\partial T / \partial z\rangle_{A, t}$ with averaging over the bottom and top plate area and in time; and (ii) a volume and time average of the conductive and convective fluxes $\mathrm{Nu}$ $=\left\langle\partial T / \partial z-(\sigma \mathrm{Ra})^{1 / 2} w T\right\rangle_{V, t}$. The dimensionless dissipations are given by $\epsilon=(\sigma / \mathrm{Ra})^{1 / 2}|\nabla \mathbf{u}|^{2}$ and $N=|\nabla T|^{2} /(\sigma \mathrm{Ra})^{1 / 2}$. These dissipations are azimuthally averaged as well as in time; they are calculated as a function of the radial and axial coordinates. It must be noted that this averaging ignores all effects that the so-called large-scale circulation (see Sec. V) could have; to reach a satisfactory convergence of the dissipation rates, azimuthal averaging is necessary. The duration of the averaging was at least $400 \tau$ for all parameter values. This amounts to roughly $200 \tau_{L}$, with $\tau_{L} \approx 2 H / U$ the large-eddyturnover time based on the circulation time of a fluid particle moving with a velocity of order $U$ along an elliptical trajectory inside the cell (cf. the large-scale circulation of Sec. V).

Furthermore, several numerical probes are distributed along two line segments. The probes are positioned at grid points and record the evolution of the three velocity components and temperature at these grid points in time. One line segment, with 74 probes on it, is on the cylinder axis from $z=0.25$ to $z=0.75$. The other, with 118 probes, is a straight line segment at half-height from $\rho=0.25, \theta=\pi$ via $\rho=0$ to $\rho=0.25, \theta=0$. The probe data are used for calculation of the SFs.

The parameters and resolution settings of the simulations are summarized in Table I. It should be noted that the grid spacing in the axial and radial directions is not uniform. Close to the walls the grid is denser in order to adequately resolve the thin viscous and thermal boundary layers. The simulations are performed at different $\mathrm{Ra}$ to investigate the dependence of $L_{B}$ and $\eta$ on Ra. Also a check for the influence of $\sigma$ has been done to be able to relate these results to the experimental data.

The accuracy of the numerical results has been validated in several ways.

First, as was mentioned in Ref. [29] the two methods for the calculation of $\mathrm{Nu}$ provide an opportunity to test the grid requirements. Method (i) mentioned above is sensitive to ad-
TABLE II. Global estimates $\langle\eta\rangle$ and $\left\langle L_{B}\right\rangle$ based on $\mathrm{Nu}$, as calculated from Eqs. (5) and (4), respectively.

\begin{tabular}{lcccc}
\hline \hline $\mathrm{Ra}$ & $\sigma$ & $\mathrm{Nu}$ & $\langle\eta\rangle$ & $\left\langle L_{B}\right\rangle$ \\
\hline $1 \times 10^{8}$ & 4 & $32.9 \pm 1.7$ & $8.35 \times 10^{-3}$ & $4.06 \times 10^{-2}$ \\
$2 \times 10^{8}$ & 4 & $40.9 \pm 1.9$ & $6.65 \times 10^{-3}$ & $3.80 \times 10^{-2}$ \\
$5 \times 10^{8}$ & 4 & $54.9 \pm 2.2$ & $4.91 \times 10^{-3}$ & $3.50 \times 10^{-2}$ \\
$1 \times 10^{9}$ & 4 & $70.9 \pm 2.7$ & $3.88 \times 10^{-3}$ & $3.35 \times 10^{-2}$ \\
$5 \times 10^{9}$ & 4 & $122.0 \pm 4.6$ & $2.26 \times 10^{-3}$ & $2.94 \times 10^{-2}$ \\
$1 \times 10^{10}$ & 4 & $163.1 \pm 5.0$ & $1.77 \times 10^{-3}$ & $2.86 \times 10^{-2}$ \\
$1 \times 10^{8}$ & 6.4 & $33.0 \pm 1.4$ & $10.56 \times 10^{-3}$ & $3.61 \times 10^{-2}$ \\
$2 \times 10^{8}$ & 6.4 & $40.8 \pm 1.7$ & $8.42 \times 10^{-3}$ & $3.38 \times 10^{-2}$ \\
$5 \times 10^{8}$ & 6.4 & $54.8 \pm 2.5$ & $6.22 \times 10^{-3}$ & $3.11 \times 10^{-2}$ \\
$1 \times 10^{9}$ & 6.4 & $71.5 \pm 2.6$ & $4.89 \times 10^{-3}$ & $2.99 \times 10^{-2}$ \\
\hline \hline
\end{tabular}

equate resolution in the boundary layers while method (ii) feels mostly the resolution in the bulk. When both methods converge to the same value, the resolution is considered sufficient. This has been verified for all simulations. The difference between the two results was always less than $1 \%$.

Second, the grid spacings have been a posteriori compared to the dissipation scales: i.e., the Kolmogorov scale $\eta$ and the Batchelor scale $\eta_{T}=\eta /\left(\sigma^{1 / 2}\right)$. In the most demanding simulation $\left(\mathrm{Ra}=1 \times 10^{10}, \sigma=4\right)$ the values found for these scales in the bulk are $\eta \approx 2 \times 10^{-3}, \eta_{T} \approx 1 \times 10^{-3}$ (Sec. IV). The largest grid-point separation in the bulk is $\Delta z=4.1$ $\times 10^{-3}$. The resolution criterion for the bulk, $\Delta z \lesssim 4 \eta_{T}$, found to be adequate in Refs. [29,31], is thus fulfilled. Near the walls, the thermal boundary layer (which is thinner than the viscous boundary layer as $\sigma>1$ ) is most sensitive to resolution. A good estimate of its thickness is $\delta_{T}=1 /(2 \mathrm{Nu})=3.1$ $\times 10^{-3}(\mathrm{Nu}$ value taken from Table II). There are 13 grid points found within the thermal boundary layer, so it is wellresolved.

Third, the simulation with $\mathrm{Ra}=1 \times 10^{10}, \sigma=4$ was repeated using a refined resolution of $257 \times 513 \times 513$. The results are similar to the results from the run at the original resolution. In particular, the Nusselt number was $161.4 \pm 5.6$ while the original result was $\mathrm{Nu}=163.1 \pm 5.0$. In view of the similarity of the results the resolution appears adequate.

\section{NUMERICAL RESULTS}

The first part of this section considers the turbulent length scales and their dependence on the position inside the cylinder. Then the SFs calculated from the simulations will be discussed.

It is illustrative for the discussion of the turbulent length scales to first get an impression of the spatial distributions of the dissipations $\epsilon$ and $N$ inside the domain. These are therefore plotted in Fig. 1 for the simulation at $\mathrm{Ra}=1 \times 10^{9}, \sigma$ $=6.4$. In the other simulations the dissipation rates are similarly distributed. It is very difficult to reliably calculate the dissipation rates near the cylinder axis owing to the metric factors $1 / \rho, 1 / \rho^{2}, \ldots$ that amplify the numerical errors in the squared gradients. For this reason we present only the values 

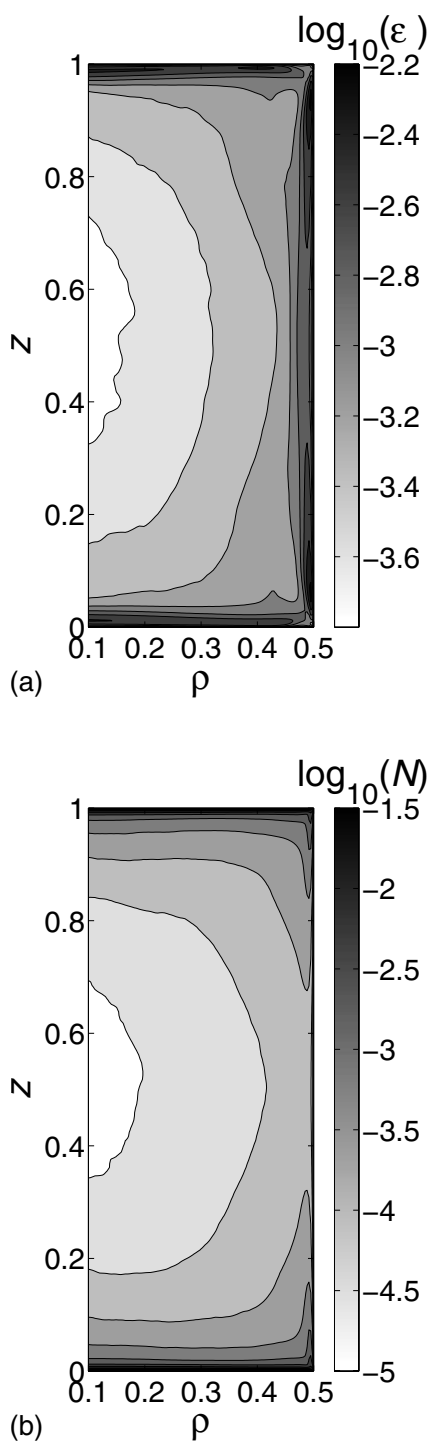

FIG. 1. Averaged dissipation rates for $\mathrm{Ra}=1 \times 10^{9}, \sigma=6.4$. The left edge of the pictures is near the cylinder axis; the right edge is the cylinder sidewall. (a) Logarithm of kinetic energy dissipation rate $\epsilon$, contour increment 0.2. (b) Logarithm of thermal variance dissipation rate $N$, contour increment 0.5 .

for $0.1 \leq \rho \leq 0.5$. This, however, is only a minor problem since as shown in Figs. 1 and 2 the isolines of all quantities tend to become orthogonal to the axis as $\rho \rightarrow 0$ (thus implying that the inner core of the flow is indeed homogeneous) and the value of each variable at the axis, if needed, can be easily extrapolated from the off-axis regions. The kinetic energy dissipation rate $\epsilon$ [Fig. 1(a)] has its maximal values inside the viscous boundary layers at the bottom and top plates, as well as on the sidewall. The temperature variance dissipation rate $N$ [Fig. 1(b)] is also high inside the (very thin) thermal boundary layers near the bottom and top plates. However, as the sidewall is adiabatic, no thermal boundary layer is present there, and $N$ shows no strong boundary layer behavior.

The turbulent length scales are, with the current dimensionless units, calculated as follows: $\eta=\sigma^{3 / 8} /\left(\mathrm{Ra}^{3 / 8} \epsilon^{1 / 4}\right), L_{B}$
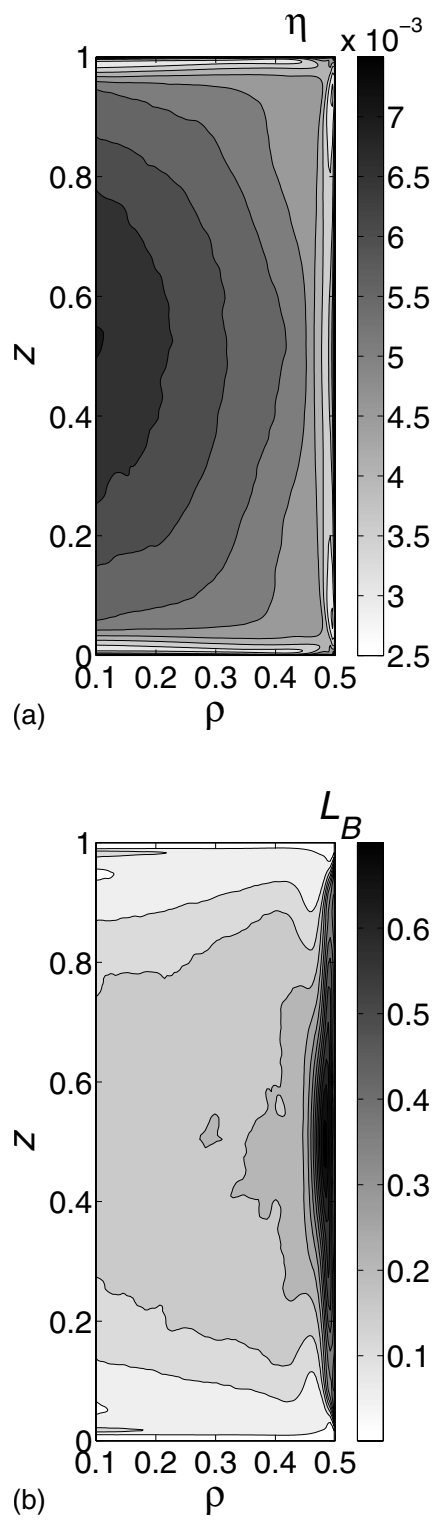

FIG. 2. Averaged length scales for $\mathrm{Ra}=1 \times 10^{9}, \sigma=6.4$. The left edge of the pictures is near the cylinder axis; the right edge is the cylinder sidewall. (a) Kolmogorov length $\eta$, contour increment $0.5 \times 10^{-3}$. (b) Bolgiano length $L_{B}$, contour increment 0.05 .

$=\epsilon^{5 / 4} / N^{3 / 4}$. These lengths are depicted in Fig. 2. The Kolmogorov length $\eta$ has the expected distribution, in that it is small inside the viscous boundary layers and attains its maximal value in the center; see Fig. 2(a). The Bolgiano length in Fig. 2(b) has a more complex distribution. Very close to the bottom and top walls, inside the thermal boundary layer, $L_{B}$ becomes very small. Just outside this region, but still inside the viscous boundary layer near the bottom and top walls, there is a local maximum of $L_{B}$ [see also the inset of Fig. 2(b)]. Traversing the domain vertically, it then gradually increases toward a roughly constant value $L_{B} \approx 0.2$ across the bulk. One additional interesting point is that $L_{B}$ has its global maximum near the sidewall, due to the presence of a viscous boundary layer (large $\epsilon$ ) while a thermal boundary layer is absent $($ small $N)$. 

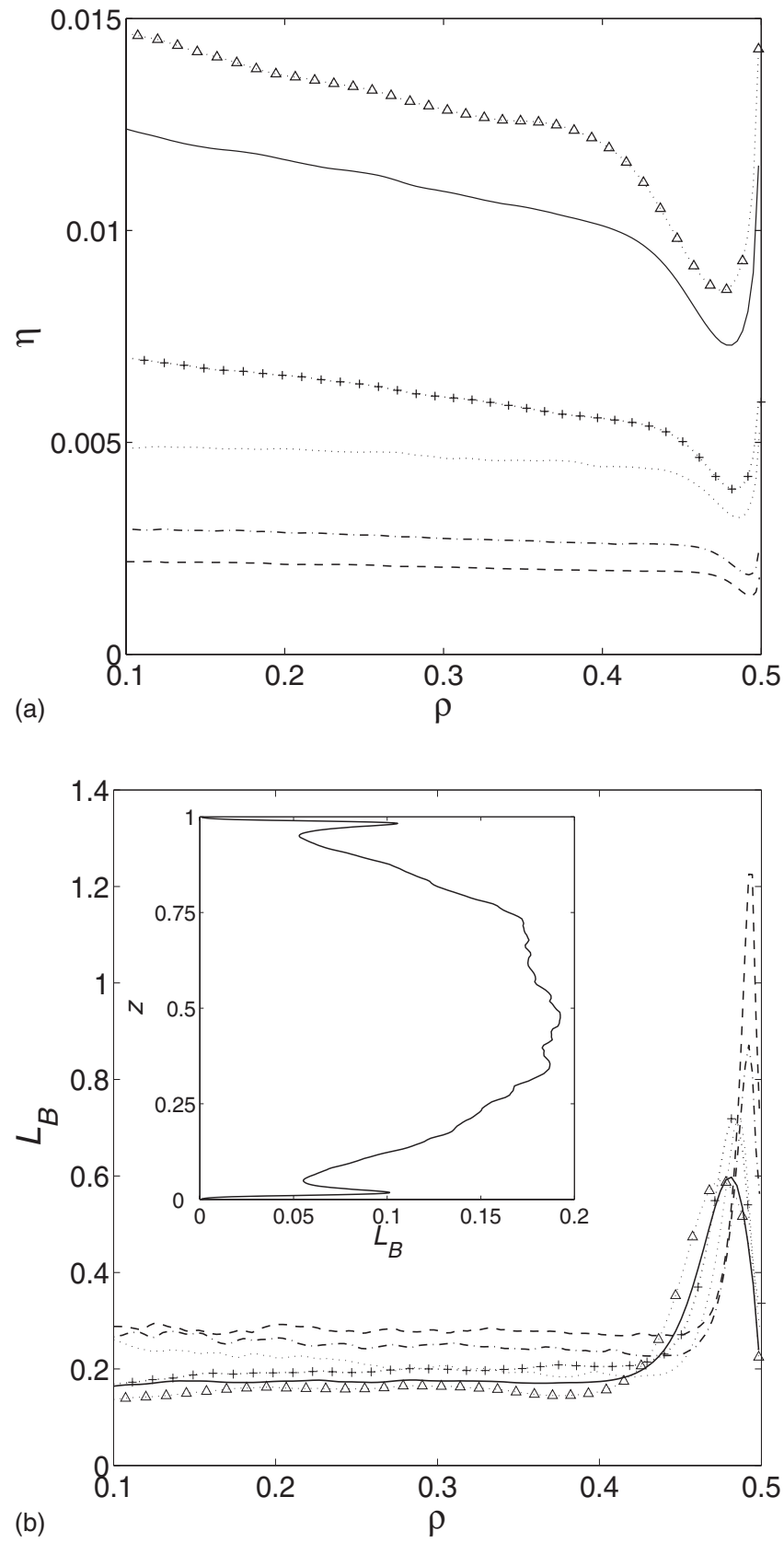

FIG. 3. Length scales at half-height as a function of radial coordinate. (a) Kolmogorov length $\eta$. (b) Bolgiano length $L_{B}$. Solid line, $\mathrm{Ra}=1 \times 10^{8}, \sigma=4$; dotted line, $\mathrm{Ra}=1 \times 10^{9}, \sigma=4$; dash-dotted line, $\mathrm{Ra}=5 \times 10^{9}, \sigma=4$; dashed line, $\mathrm{Ra}=1 \times 10^{10}, \sigma=4$; dotted line with triangles, $\mathrm{Ra}=1 \times 10^{8}, \sigma=6.4$; dotted line with pluses, $\mathrm{Ra}=1$ $\times 10^{9}, \sigma=6.4$. Some simulation results are left out for clarity. The inset in (b) shows $L_{B}$ as a function of the axial coordinate $z$ ( $\rho$ $=0.2$ ) for the case $\mathrm{Ra}=1 \times 10^{9}, \sigma=6.4$.

To compare the length scales obtained from the simulations we present their values along a horizontal cross section at half-height in Fig. 3. Also, the lengths as calculated from the global averages are given in Table II. In Fig. 3(a) it is found that $\eta$ decreases monotonically in the bulk toward the sidewall and shows a minimum within the viscous boundary layer. At higher Ra, $\eta$ is smaller, as is expected for a flow with a higher turbulence intensity. Note also the differences

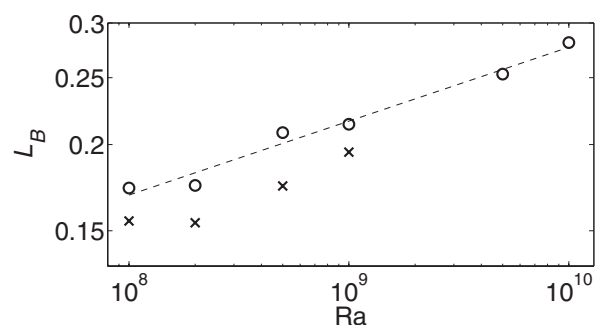

FIG. 4. Local values of $L_{B}$ as a function of Ra. The circles are taken from the $\sigma=4$ simulations, while the crosses indicate the $\sigma$ $=6.4$ results. The dashed line is the power-law fit to the circles given in Eq. (7).

as a result of changing $\sigma$ for the runs with $\mathrm{Ra}=1 \times 10^{8}$ and $\mathrm{Ra}=1 \times 10^{9}$ (dotted line compared to dotted line with pluses and solid line compared to dotted line with triangles, respectively). An increase in $\sigma$ yields a larger $\eta$. The globalaverage values $\langle\eta\rangle$ from Table II show rather good compliance with the local values at half-height (within a factor of 2).

Continuing to Fig. 3(b), $L_{B}$ is found to be constant in the bulk region. A comparison with the global-average values shows that the local bulk values can be about one order of magnitude larger. Furthermore, the global-average $\left\langle L_{B}\right\rangle$ values from Table II decrease when Ra increases, while its local value actually increases. These points indicate that the oftenused formula (4) for $\left\langle L_{B}\right\rangle$ is not suitable for the interpretation of SF results, since the estimate can be off by an order of magnitude compared to the actual value. Concluding, for resolving a possible $\mathrm{BO}$ regime one must investigate scales much larger than the estimate of Eq. (4). A decreased bulk $L_{B}$ value can be achieved by lowering $\mathrm{Ra}$.

From the inset of Fig. 3(b) it becomes clear that $L_{B}$ is also smaller when approaching the bottom or top plates, but outside of the boundary layers. This observation was also reported in Refs. [19,29,32]. Hence, observation of the BO scaling regime is easier when measuring outside of the central part of the cylinder as $L_{B}$ is smaller there.

The values for $L_{B}$, averaged over $0.1<\rho<0.4$ at halfheight, are shown as a function of Ra in Fig. 4. A power-law fit is given by

$$
L_{B}=0.024 \mathrm{Ra}^{0.107 \pm 0.016}
$$

The slope of this fit matches also with the $\sigma=6.4$ points (crosses), but with a slight downward shift. However, there may be two different regimes to be identified. In the presentation of the experimental SF results in Sec. VI these simulation results (represented by the crosses) will be used to estimate the value of $L_{B}$ at the center of the cell.

From the simulation results it is possible to calculate both velocity and temperature SFs. We adopt the following steps. Since the grid is nonuniform in radial as well as in axial directions, the numerical probe data were first interpolated onto a uniform grid with a cubic spline interpolation algorithm separately for each time step. Then, direct calculation of the velocity differences as a function of the separation, followed by time averaging, gave the SF results. 

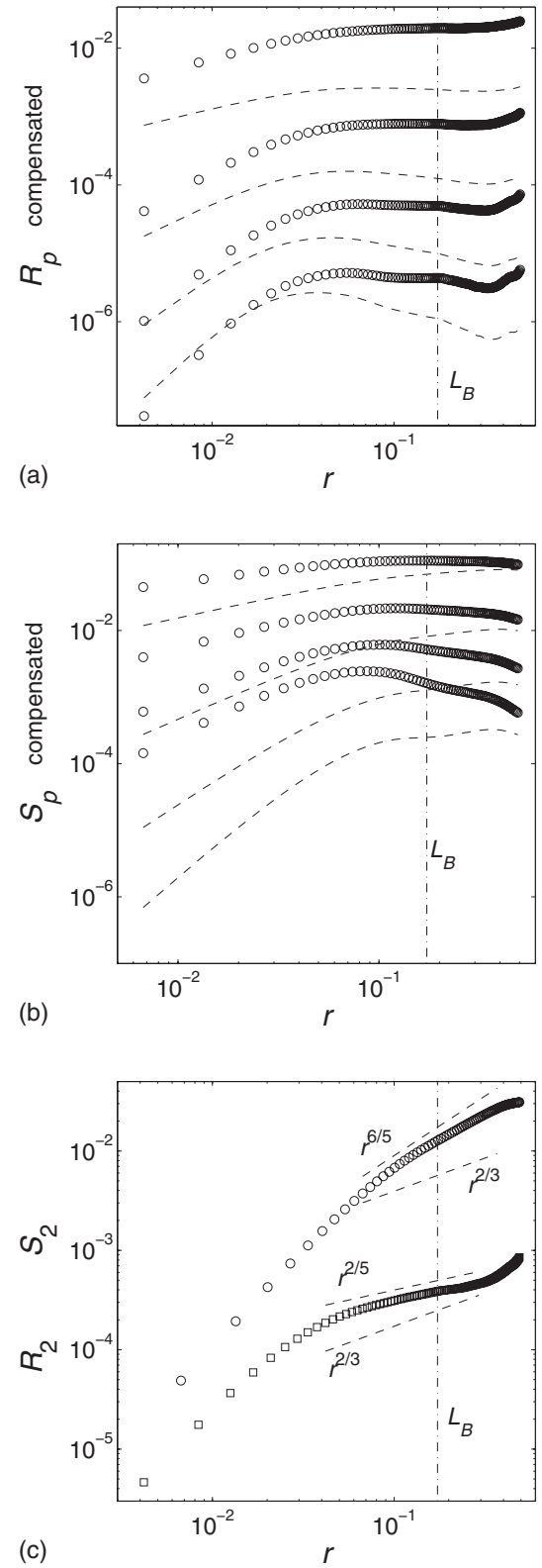

FIG. 5. Compensated structure functions taken at $\mathrm{Ra}=1 \times 10^{8}$, $\sigma=4$ of (a) temperature calculated in the radial direction and (b) vertical velocity calculated in the axial direction. The circles denote the BO-compensated SFs, while the dashed lines indicate the OCcompensated SFs. A downward shift of one decade is used for the OC-compensated temperature SFs for clarity. The order $p$ increases from 1 to 4 from top to bottom. The local Bolgiano length $L_{B}$ is indicated with the dash-dotted line. (c) The second-order SFs of (a) and (b) plotted without compensation: $R_{2}$ (squares) and $S_{2}$ (circles, shifted upward by half a decade), with reference slopes (dashed lines).

Since a smaller $L_{B}$ allows for a larger range of scales above $L_{B}$, we start the discussion of the SFs at the lowest Rayleigh number considered, $\mathrm{Ra}=10^{8}$, with correspondingly the smallest $L_{B}$ (cf. Fig. 4). For the case $\mathrm{Ra}=1 \times 10^{8}, \sigma=4$, the temperature structure functions up to order 4 are shown in Fig. 5(a) as calculated along the radial direction at midheight. They are compensated for BO scaling (circles) and

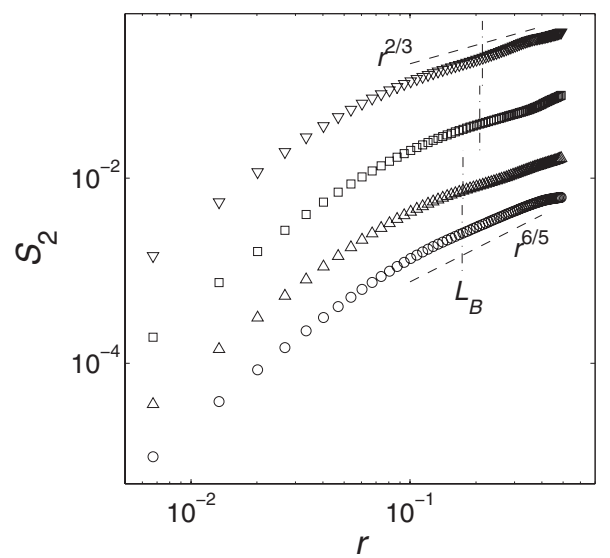

FIG. 6. Second-order SFs of vertical velocity calculated in axial direction, for $\sigma=4$ and $\mathrm{Ra}=1 \times 10^{8}$ (circles), $\mathrm{Ra}=2 \times 10^{8}$ (up triangles), $\mathrm{Ra}=5 \times 10^{8}$ (squares), and $\mathrm{Ra}=1 \times 10^{9}$ (down triangles). The four curves are shifted apart for clarity. The Bolgiano length $L_{B}$, taken from Fig. 4 , is included for each Ra (dash-dotted lines). Dashed lines are reference slopes.

for OC scaling (dashed line). Also indicated (dash-dotted line) is the Bolgiano length observed in the central region, taken from Fig. 3(b). It is found that BO scaling is more appropriate for a range of $r$ around $r=L_{B}$. Indeed, the OCcompensated graphs show no plateau, just a negative slope. The $L_{B}$ estimate does not exactly indicate the beginning of the scaling range; it is roughly a factor of 2 larger. This is not surprising given that it is a dimensional estimate.

The vertical-velocity SFs calculated in the radial direction exhibit neither BO nor K41 scaling; they show a gradual transition from the $\sim r^{p}$ behavior expected in the viscous subrange to a plateau at the transverse integral length scale $L_{\perp}$ (see, e.g., Ref. [33]). However, when the velocity SFs are calculated in the axial direction the $\mathrm{BO}$ scaling range can be recognized again. In Fig. 5(b) the SFs of vertical velocity calculated in the axial direction are shown, compensated by both BO and K41 scalings. The local Bolgiano length becomes smaller outside of the central region of the cylinder, but not by much in the range $0.25<z<0.75$; see the inset of Fig. 3(b). But since now we consider a longitudinal SF, the longitudinal integral length scale $L_{\|}$is found to be larger (for isotropic turbulence this can be quantified: $L_{\|}=2 L_{\perp}$ [33]). Thus a longer scaling range is present between $L_{B}$ and $L_{\|}$, compared to its transverse counterpart, making its detection easier. It can also be seen that as $p$ increases there is a gradual deviation from pure BO scaling which we suspect is due to intermittency. As found in Fig. 5(c) the second-order SFs show the predicted BO slopes.

Next, we consider the effect of different $\mathrm{Ra}$ on the SF scaling. The second-order SFs of vertical velocity calculated in the axial direction are depicted in Fig. 6 at several $\mathrm{Ra}$ in the range $1 \times 10^{8} \leq \mathrm{Ra} \leq 1 \times 10^{9}$ and Prandtl number $\sigma=4$. Starting with the lowest curve in Fig. 6, corresponding to the lowest $\mathrm{Ra}=1 \times 10^{8}$, the SF exhibits BO scaling. This changes into SFs, which display, respectively, an intermediate slope, a separation into two ranges (K41 and BO) observed from compensated plots (not shown here), and finally an only-K41 $\mathrm{SF}$ as $\mathrm{Ra}$ increases. A more detailed impression of this tran- 


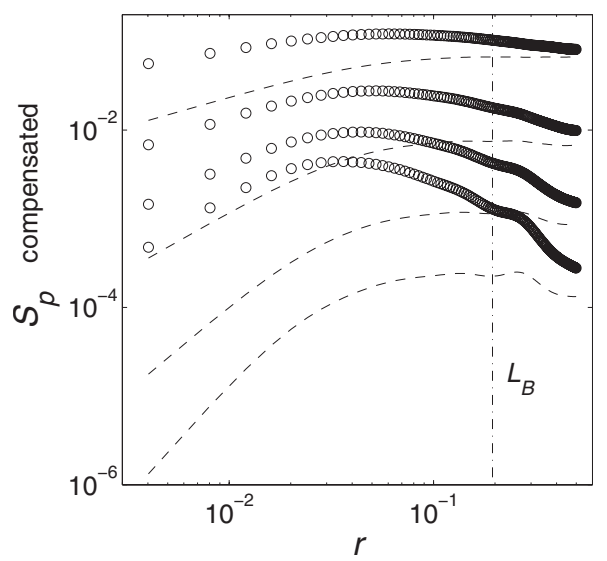

FIG. 7. Compensated structure functions of vertical velocity calculated in the radial direction at $\mathrm{Ra}=1 \times 10^{9}, \sigma=6.4$. The circles denote the BO-compensated SFs, while the dashed lines indicate the K41-compensated SFs. The order $p$ increases from 1 to 4 from top to bottom. The local Bolgiano length $L_{B}$ is indicated by the dashdotted line.

sition is difficult to obtain with the current simulation approach, since the separations between the three length scales involved (Kolmogorov length $\eta$, Bolgiano length $L_{B}$, and the integral length scale $L$ ) remain rather small. Therefore, the scaling ranges are quite small or are somewhat smeared out in the transition to neighboring ranges; cf. the case $\mathrm{Ra}=2$ $\times 10^{8}$ in Fig. 6 (up triangles). A larger-aspect-ratio cylinder with increased resolution might better separate the length scales, but to achieve similar Rayleigh numbers would then require considerably more computational resources.

In all other simulations only a K41 scaling range is observed. As an example, and for comparison with the experiments, the SFs of the vertical velocity calculated for the radial direction at $\mathrm{Ra}=1 \times 10^{9}$ and $\sigma=6.4$ are depicted in Fig. 7 , again K41 and BO compensated. Here, clearly, K41 is the valid scaling. The local Bolgiano length is larger than for the previous case. In the SF plots there is a change of slope visible above $L_{B}$; however, the very small range of $r>L_{B}$ prevents any definitive conclusion on the change of slope. Still, the temperature SFs show BO scaling similar to Fig. 5(a). An analysis using extended self-similarity (ESS) [34] can provide more insight into this scaling; we discuss these results further in Sec. VI.

\section{EXPERIMENTAL ARRANGEMENT AND VALIDATION}

Our convection cell is depicted schematically in Fig. 8. Letters between brackets in this paragraph and the next refer to labels in this sketch. The cell consists of a Plexiglas cylinder (A) of diameter and height $230 \mathrm{~mm}$ (aspect ratio 1). It is closed from below with a 30-mm-thick copper plate (B) with a resistance heater $(\mathrm{C})$ connected underneath and closed from above with a thin (1-mm) layer of Plexiglas (D). Mounted above the cylinder is a transparent cooling chamber (E) through which water is circulated that is kept at a constant temperature by an external cooling bath. There are four tubes connected on either side of this chamber. Inside the

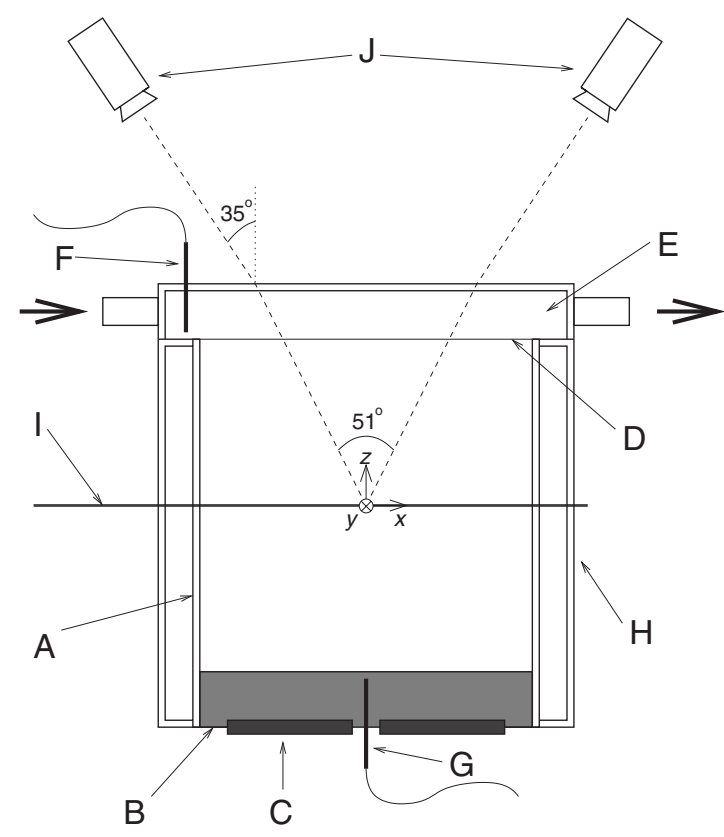

FIG. 8. Schematic cross-sectional view of the convection cell (not to scale). Labels are explained in the text. The coordinates are defined with respect to the center of the cell.

tubes finely meshed grids are placed to induce turbulence in the flow inside the cooling chamber to avoid large-scale flow patterns there. A considerable flow of cooling water through the chamber ensures constant-temperature conditions at the top of the cylinder. This transparent construction is necessary for optical access from above. A Pt-1000 temperature sensor inside the cooling chamber (F) is used as the feedback sensor for the cooling bath. It shows maximal deviations of $\pm 0.04{ }^{\circ} \mathrm{C}$ from the preset temperature (with $\Delta T$ in the range $0.5-5{ }^{\circ} \mathrm{C}$ ). The temperature inside the cooling chamber has been measured at several positions within the cooling chamber; the temperature was found to be uniform within measurement precision. Another temperature sensor $(\mathrm{G})$ is placed inside a hole in the copper plate close to the surface that is in contact with the water. The heater controller keeps the temperature of the copper plate constant up to $\pm 0.02{ }^{\circ} \mathrm{C}$. The side of the cylinder is surrounded by a square Plexiglas box (H). The space in between the square box and the outer side of the cylinder is also filled with water to facilitate optical access from the side. It should be mentioned that the current setup has parasitic heat leakage through the surrounding box. However, the parasitic heat flux is expected to have only very minor influence on the actual flow inside the container, so for the purpose of this experiment (measurement of flow statistics) it is of little consequence.

The water inside the cylinder is seeded with Dantec polyamid seeding particles of diameter $50 \mu \mathrm{m}$. The particles are nearly neutrally buoyant (density $1.03 \mathrm{~g} / \mathrm{cm}^{3}$ ) and are small enough to not disturb the flow. A pulsed Nd:YAG laser operated at $15 \mathrm{~Hz}$ provides a $\approx 2$-mm-thick light sheet (I) that intersects the cylinder horizontally at half-height or at 45 $\mathrm{mm}$ below the top plate. Two charge-coupled-device (CCD) video cameras (J) (1 megapixel, 10-bit dynamic range), mounted above the cylinder with a common angle ("angular 
displacement" configuration [26]), record the reflected light from the particles. Off-angle placement of the cameras allows for detection of vertical displacements in addition to horizontal movement, but necessitates the use of camera lens adapters to fulfill the Scheimpflug condition [26]. The stereoscopic angle inside the cell is $51^{\circ}$. Raw images are processed with an advanced SPIV algorithm with misalignment correction [35]. The result is a two-dimensional threecomponent velocity field in the light sheet.

From the findings of Sec. IV concerning the length scales it can be concluded that a large measurement area is required, since the Bolgiano length $L_{B}$ covers already roughly one-fourth of the cylinder extent. In this work, the actual measurement area (the common area in the camera images) was roughly $90 \times 120 \mathrm{~mm}^{2}$ for the measurements at halfheight and $120 \times 150 \mathrm{~mm}^{2}$ for the measurements closer to the top lid. Since a stereoscopic angle is essential for the measurement technique, it is not feasible to measure on the entire circular cross-section of the cylinder simultaneously. The interrogation windows used were $32 \times 32$ pixels in size for the half-height experiments and $16 \times 16$ pixels for the experiments closer to the top lid. A lower mean vertical velocity along with a shorter optical path through the water (more scattered light from the particles is captured) made it possible to decrease the window size for the experiments near the top lid. A 50\% overlap between neighboring interrogation windows is used. After processing, the velocity fields consist of $49 \times 57$ vectors on a uniform grid with spacings $\Delta x=1.66 \mathrm{~mm}$ and $\Delta y=1.92 \mathrm{~mm}$ for the half-height experiments. From the experiments near the top wall the velocity fields have $107 \times 111$ vectors with spacings $\Delta x$ $=1.15 \mathrm{~mm}$ and $\Delta y=1.39 \mathrm{~mm}$. The total number of vectors is considerably smaller than for PIV studies with a camera of similar resolution and interrogation window size. Only the field of view common to both cameras is used in SPIV; this is only a part of the actual field of view of a single camera. In this case about $75 \%$ of the field of view per camera is used, compared to (up to) $100 \%$ in PIV.

In the experiments presented here the average temperature of the water was $24.0^{\circ} \mathrm{C}$. Under these conditions $\sigma=6.37$. The three temperature differences used were $0.5^{\circ} \mathrm{C}, 1.5^{\circ} \mathrm{C}$, and $5^{\circ} \mathrm{C}$, corresponding to $\mathrm{Ra}=1.11 \times 10^{8}, 3.34 \times 10^{8}$, and $1.11 \times 10^{9}$, respectively. In the half-height experiments velocity fields are taken in series of about $10^{4}$ snapshots at 15 $\mathrm{Hz}$, the measurement duration being just over $11 \mathrm{~min}$. For the experiments closer to the top lid a different approach was chosen: each camera recorded two subsequent snapshots, separated in time by $1 / 15 \mathrm{~s}$. One couple of subsequent snapshots was recorded per second so that in the processing one velocity field per second could be calculated. A total of 4 $\times 10^{3}$ snapshot pairs (and thus also velocity fields) per experiment were taken for a measurement duration just over 1 $\mathrm{h}$. This longer time scale was necessary for the analysis of the large-scale circulation of confined convection, as will become clear from what follows next.

Many studies on turbulent convection focus on the observed structuring of the flow into a domain-filling circulation driven by the cold and hot thermal plumes erupting from the thermal boundary layers on the top and bottom walls. The cold sinking plumes tend to gather on one side of the
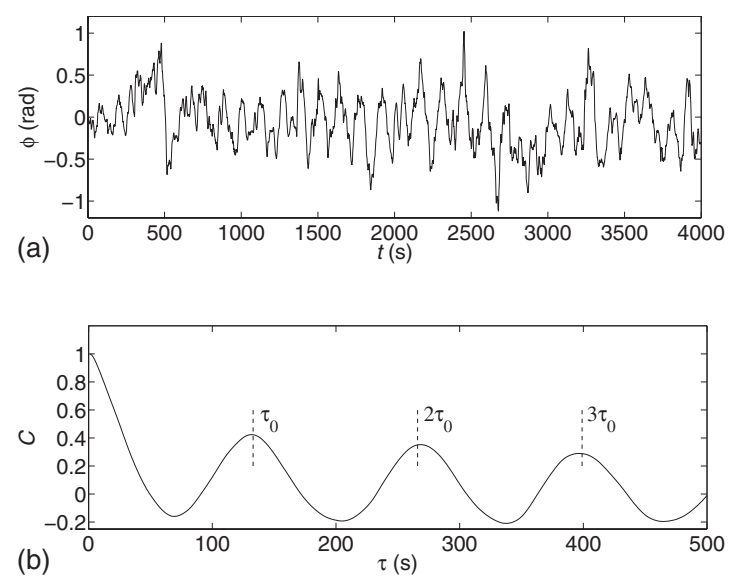

FIG. 9. Investigation of the LSC orientation at $\mathrm{Ra}=1.11 \times 10^{9}$. (a) Orientation $\phi$ as a function of time. (b) Autocorrelation $C(\tau)$ of $\phi$. The oscillation period $\tau_{0}$ and secondary peaks are labeled.

tank, while hot rising plumes gather on the opposite side. This general motion is known as large-scale circulation. The LSC is known to be active on many different time scales, from an azimuthal oscillation with a time scale of the order of $1 \mathrm{~min}$ (for convection cells of similar dimension to the current cell) to so-called rotations and cessations that occur irregularly with intervals of hours or even days. Recent works include [27,36,37].

Here we present an investigation of the azimuthal oscillation of the LSC to compare the performance of the current setup with others. To this end the velocity data of the "high" cross-section are used. To find the azimuthal orientation of the LSC we use an idea from [27]. The orientation $\phi$ of the LSC is defined as the orientation of the (horizontal) vector $\mathbf{U}=\langle u\rangle \hat{\mathbf{x}}+\langle v\rangle \hat{\mathbf{y}}$. Here the angular brackets indicate spatial averaging over all vectors within a circular area centered at the center of the measurement area, the diameter of the circular area being equal to the $x$ extent of the rectangular measurement area (which is smaller than the $y$ extent). Inside this circular region there are 7314 vectors for averaging. The evolution of $\phi$ in time is recorded and an autocorrelation of this series gives the average oscillation period $\tau_{0}$. As an example, for the experiment at $\mathrm{Ra}=1.11 \times 10^{9}$, in Fig. 9(a) we plot the evolution of the LSC orientation $\phi$ in time, while Fig. 9(b) holds the autocorrelation $C(\tau)$ as a function of the time gap $\tau$. The oscillation period is recognized as peaks in $C(\tau)$, with the peaks at $\tau_{0}, 2 \tau_{0}$, and $3 \tau_{0}$ labeled. In this case $\tau_{0}=133 \pm 2 \mathrm{~s}$, where the maxima are found by second-order polynomial fitting of the peak regions and the first 20 peak locations are calculated to estimate the spread. When the oscillation frequency is made dimensionless as $f_{0}$ $=H^{2} /\left(\kappa \tau_{0}\right)$, we arrive at $f_{0}=(2.74 \pm 0.04) \times 10^{3}$. This value is consistent with the experimentally obtained correlations $f_{0}$ $=0.084 \mathrm{Ra}^{0.50 \pm 0.01}$ from Ref. [27] and $f_{0}=0.201 \mathrm{Ra}^{0.460 \pm 0.012}$ from Ref. [38].

The experiments at the other Rayleigh numbers are treated similarly. In the $\mathrm{Ra}=3.34 \times 10^{8}$ experiment this gave $\tau_{0}=212 \pm 3 \mathrm{~s}$, based on 15 peaks of $C(\tau)$. This corresponds to $f_{0}=(1.72 \pm 0.02) \times 10^{3}$, also consistent with Ref. $[27,38]$. In the $\mathrm{Ra}=1.11 \times 10^{8}$ experiment there were no clear peaks 


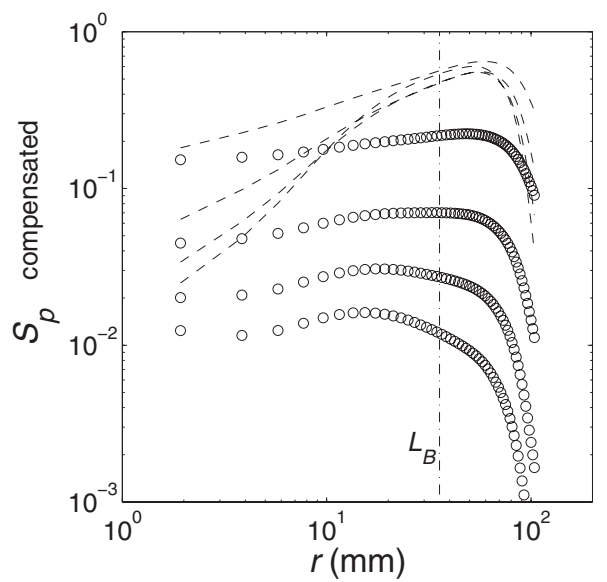

FIG. 10. Compensated SFs of $w$ calculated along $y$ for Ra $=1.11 \times 10^{8}$. Circles are for BO-compensated SFs, while dashed lines indicate K41-compensated SFs. The order $p$ increases from 1 to 4 from top to bottom on the left-hand side of the graph. The estimated $L_{B}$ is given by the dash-dotted line.

in the autocorrelation graph. At lower Rayleigh number the LSC is less intense and its oscillation period longer. The method used here to detect the LSC is not conclusive in this case. Also, this $\mathrm{Ra}$ value is close to the critical value $\mathrm{Ra}_{c}$ for the formation of an LSC $\left(\mathrm{Ra}_{c} \approx 5 \times 10^{7}\right.$ was reported in Ref. [39] for a cell of similar dimensions). Based on the current measurement we refrain from drawing conclusions on the LSC at this value of $\mathrm{Ra}$ in our setup.

\section{EXPERIMENTAL RESULTS}

In this section we discuss the SFs calculated from the measurements taken at the cell half-height. Then extended self-similarity [34] is applied. We finally mention the difficulties we encountered while interpreting the SF results from the measurements taken closer to the top wall.

The first experimental SF result to be discussed is taken at $\mathrm{Ra}=1.11 \times 10^{8}$. It is the prime candidate for BO scaling according to the $L_{B}$ results of Fig. 4 . Of all Ra considered, $L_{B}$ is smallest in this case, so the largest range above this scale is available. An investigation of the SF scaling is done in Fig. 10 , where SFs of orders $1-4$ of $w$ are plotted, both $\mathrm{BO}$ and $\mathrm{K} 41$ compensated. Indeed $\mathrm{BO}$ is the appropriate scaling around the $L_{B}$ indication. The estimate for $L_{B}$ seems to be slightly higher than the actual value as the scaling range is also observed for smaller separations. As in the previous DNS results the actual scalings deviate rapidly from the theoretical $\sim r^{3 p / 5}$ predictions, which we suspect is due to intermittency. No K41 range is found below $L_{B}$; the resolution is insufficient at small $r$.

The scaling range is not very wide. The top end of the scaling range is the so-called inertial length. A SF should reach a plateau at a separation $r$ equal to the inertial length [33]. At larger separations the velocities are uncorrelated, yielding a constant-on-average velocity difference and hence a constant SF value. It is easily verified theoretically that the plateau of $S_{2}^{w}$ should be at $2\left\langle w^{2}\right\rangle$ [33]. This was observed for the current SF.

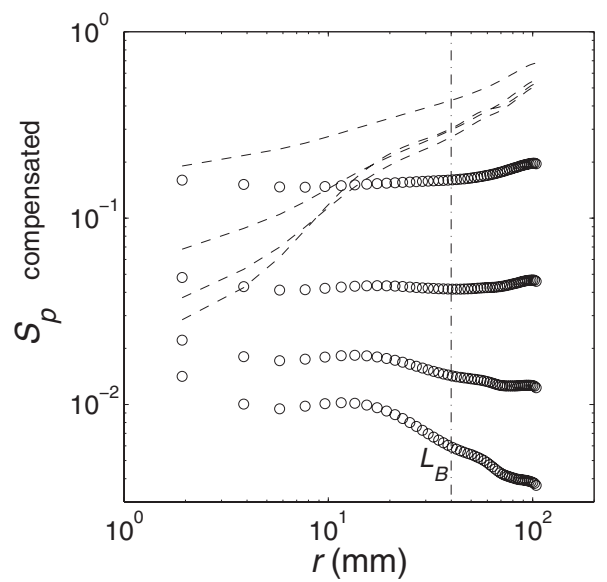

FIG. 11. Compensated SFs of $w$ calculated along $y$ for Ra $=3.34 \times 10^{8}$. Circles are for BO-compensated SFs, while dashed lines indicate K41-compensated SFs. The order $p$ increases from 1 to 4 top to bottom. The estimated $L_{B}$ is indicated by the dash-dotted line.

Continuing to the higher $\mathrm{Ra}=3.34 \times 10^{8}$, the compensated SFs of $w$ for this case are plotted in Fig. 11. The estimated $L_{B}$ is indicated by the dash-dotted line. Indeed, a BO range is found around $L_{B}$. The gradual deviation from pure $r^{3 p / 5}$ behavior is observed as $p$ increases.

For the highest Ra considered here, $\mathrm{Ra}=1.11 \times 10^{9}$, the compensated SFs of $w$ are shown in Fig. 12. Again, an even wider $\mathrm{BO}$ range is evident. The integral length scale is now larger than the measurement area; scaling continues up to the largest $r$. For a very small interval below $L_{B}$, roughly starting from $r \approx 20 \mathrm{~mm}$, there is even a slight hint of K41 scaling with a plateau in the third-order K41-compensated SF

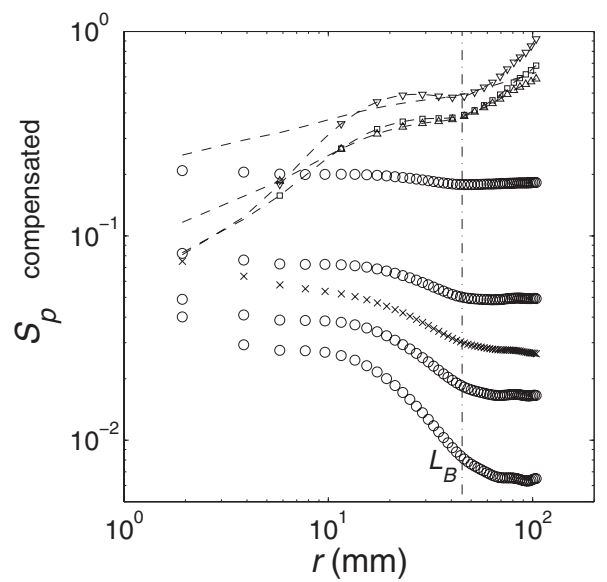

FIG. 12. Compensated SFs of $w$ calculated along $y$ for Ra $=1.11 \times 10^{9}$. Circles are for BO-compensated SFs, while dashed lines indicate K41-compensated SFs. The order $p$ increases from 1 to 4 from top to bottom for BO. The dashed line without symbols is the $p=1 \mathrm{SF}$ with K41 compensation. Similarly, dashed lines with up triangles, squares, and down triangles are for $p=2,3$, and 4 , respectively. Crosses indicate the second-order SF compensated for $r^{4 / 3}$ (this will be discussed later on). The estimated $L_{B}$ is indicated by the dash-dotted line. 


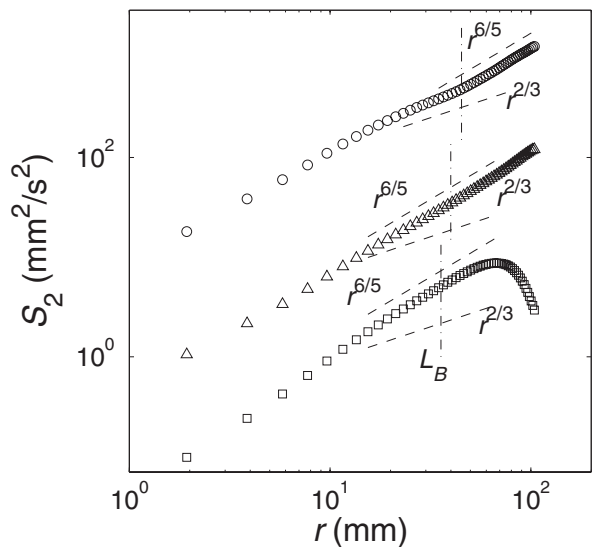

FIG. 13. Second-order SFs of $w$ for $\mathrm{Ra}=1.11 \times 10^{8}$ (squares), $\mathrm{Ra}=3.34 \times 10^{8}$ (triangles, shifted upward by one decade), and Ra $=1.11 \times 10^{9}$ (circles, shifted upward by two decades). Again, the corresponding $L_{B} \mathrm{~s}$ are indicated by the dash-dotted lines. Dashed lines are reference slopes.

(dashed line with squares). However, the current resolution does not allow us to resolve this range accurately.

As a summary of the experimental SF results, in Fig. 13 we present the second-order SFs for the three Ra values in one figure. The shift of $L_{B}$, and also the range of scaling, as a function of $\mathrm{Ra}$ is apparent.

We next directly compare the numerical SFs to the ones obtained from experiment. A comparison of cases that have indications of both $\mathrm{K} 41$ and $\mathrm{BO}$ scaling ranges is shown in Fig. 14, including the DNS results at $\mathrm{Ra}=2 \times 10^{8}$ and $\mathrm{Ra}$ $=5 \times 10^{8}$, both at $\sigma=6.4$, and the experimental result at $\mathrm{Ra}$ $=3.34 \times 10^{8}, \sigma=6.37$. On the low $r$ end, the available resolution in the experiment causes the dissipative range to be represented with lower accuracy. In the DNS the transition from the K41 scaling to the steeper scaling at higher $r$ is found at a length scale of about a factor of 2 larger than in the experiment. The differences between simulation and experiment are evident as the idealized condition represented in the numerics is compared to the experimental setup with its

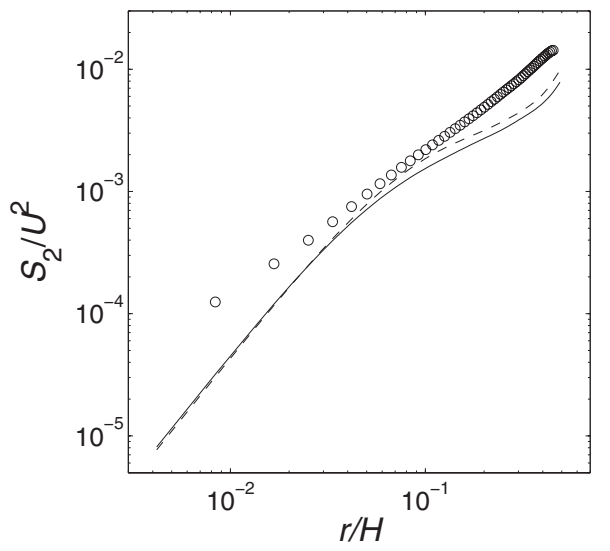

FIG. 14. Second-order SFs of vertical velocity, a comparison between simulation and experiment. Circles: experiment at $\mathrm{Ra}$ $=3.34 \times 10^{8}, \sigma=6.37$. Dashed line: simulation at $\mathrm{Ra}=2 \times 10^{8}, \sigma$ $=6.4$. Solid line: simulation at $\mathrm{Ra}=5 \times 10^{8}, \sigma=6.4$.

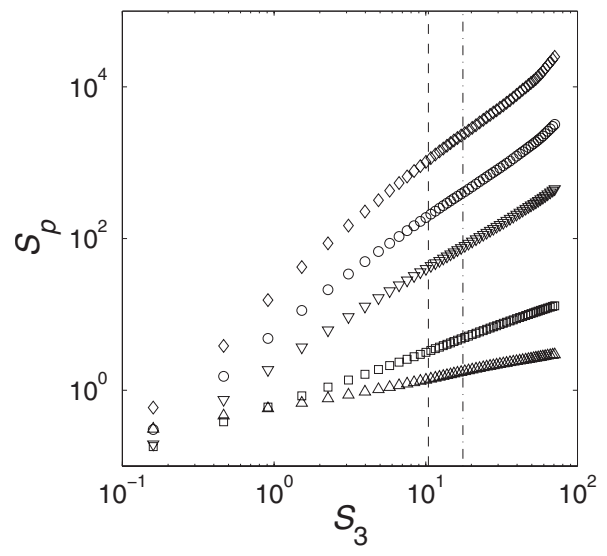

FIG. 15. Extended self-similarity plot for $\mathrm{Ra}=1.11 \times 10^{9}$. SFs of $w$ of order $p=1$ (up triangles), 2 (squares), 4 (down triangles), 5 (circles), and 6 (diamonds) are plotted as a function of the thirdorder SF of $w$. The dash-dotted line indicates the $L_{B}$ estimate. At the dashed line a slope change is found in all curves.

uncertainties and external influences. There is general agreement, and the scaling regimes are well captured by both.

A way to provide more insight into the SF behavior is to apply extended self-similarity [34]. ESS widens the range of scaling of an SF by plotting the SF of order $p$ as a function of the SF of order 3 and allows detection of a scaling range at far lower Rayleigh numbers. An ESS plot for the $\mathrm{Ra}=1.11$ $\times 10^{9}$ case, shown before in Fig. 12, is presented in Fig. 15, containing the SFs up to order $p=6$. There is a slope change found in all five curves; the position of which is indicated by the dashed line. It is found to be near, but somewhat below, the position corresponding to the Bolgiano length (the dashdotted line). Indeed, there is scaling on both sides of the dashed line. The plots for the other Ra cases are shown in Fig. 16.

Power-law fits in both regions with scaling give the exponents $\xi_{p}$, which can be conveniently plotted against the order $p$, as is done in Fig. 17. The fits of the non-BO regime (triangles, squares, and circles) coincide very well and follow the predictions of the hierarchical-shell model proposed by She and Leveque [40] (dashed line). In the BO regime (diamonds, crosses, and pluses) there is a deviation, which would indicate stronger intermittency effects. There is a larger spread of the three measurements compared with the non-BO results. It is possible that there is some $\mathrm{Ra}$ dependence to be found, with the measured result most likely somewhat contaminated by the non-BO range as $\mathrm{Ra}$ increases. It must be stressed here that $\xi_{p}$ are relative scaling exponents $S_{p}(r) \sim\left[S_{3}(r)\right]^{\xi_{p}}$ and not absolute scalings $S_{p}(r)$ $\sim r^{\zeta_{p}}$ (see, e.g., Grossmann et al. [41]), and they can only be equal if $\zeta_{3}=1$, which is clearly not valid here.

We also performed an ESS analysis for the DNS results of Sec. IV. The same trend was observed: the slopes change at a point corresponding to a scale somewhat smaller than $L_{B}$. Consistently, this point is found at larger scales for higher Ra.

It has been mentioned in Sec. IV that a BO range should be more readily measurable at vertical levels closer to the top or bottom of the cell, since there $L_{B}$ is smaller and thus an 

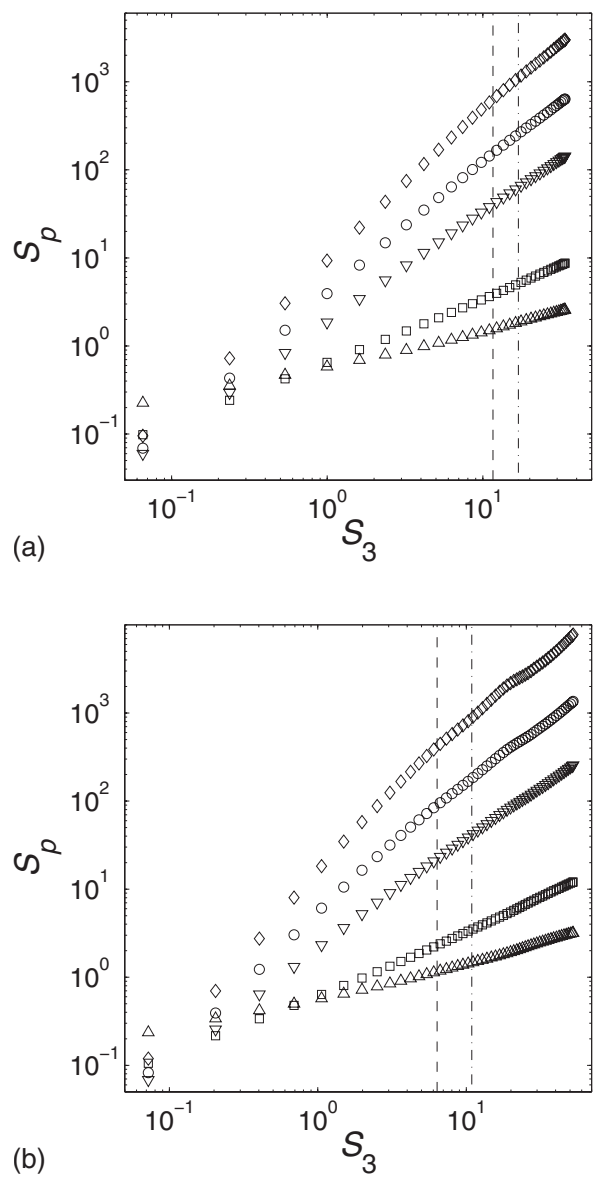

FIG. 16. (a) Same as Fig. 15 , but now for $\mathrm{Ra}=1.11 \times 10^{8}$. (b) Same as Fig. 15, but now for $\mathrm{Ra}=3.34 \times 10^{8}$.

extended range of larger scales is attainable in the measurements. Thus, SFs have also been calculated from the velocity data taken nearer to the top plate. In Fig. 18 an example plot of the second-order SFs of $w$ calculated in both $x$ and $y$ directions can be found. At a certain separation $r$ the curves diverge. This is an effect of the LSC and the separation of upward and downward motions; it is acting as a linear shear disturbance, the direction of which oscillates in time.

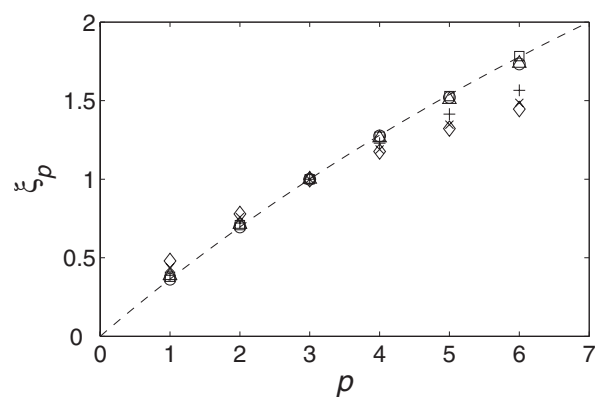

FIG. 17. Scaling exponents $\xi_{p}$ as a function of the order $p$ from the ESS plots. Non-BO range: triangles are for $\mathrm{Ra}=1.11 \times 10^{8}$, squares for $\mathrm{Ra}=3.34 \times 10^{8}$, and circles for $\mathrm{Ra}=1.11 \times 10^{9}$. BO range: diamonds are for $\mathrm{Ra}=1.11 \times 10^{8}$, crosses for $\mathrm{Ra}=3.34$ $\times 10^{8}$, and pluses for $\mathrm{Ra}=1.11 \times 10^{9}$. The dashed line indicates the hierarchical-shell model of Ref. [40].

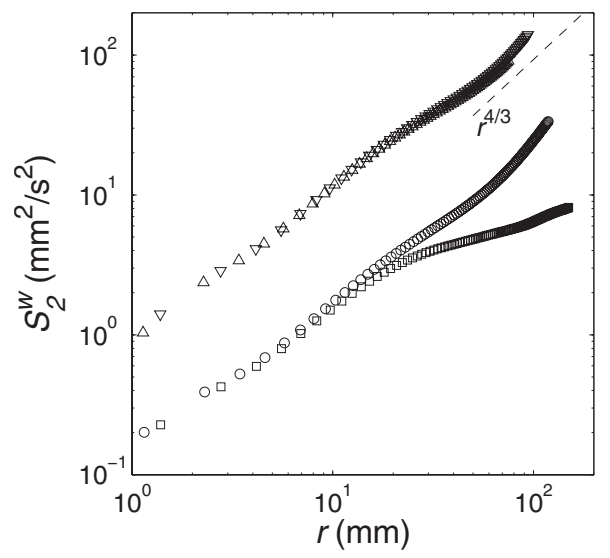

FIG. 18. The second-order structure functions of $w$, measured near the top wall at $\mathrm{Ra}=1.11 \times 10^{9}$. Before correction: circles for $S_{w}^{2}$ calculated along $x$, squares for $S_{w}^{2}$ calculated along $y$. After correction (coordinate axis rotation of $0.92 \mathrm{rad}$ ): up (down) triangles for $S_{w}^{2}$ calculated along $x(y)$. The corrected SFs are shifted up for clarity.

The situation is sketched in Fig. 19. It is known that, for homogeneous shear flow, the shear induces changes in the SFs for $r \geqslant L_{\alpha} \equiv\left(\epsilon / \alpha^{3}\right)^{1 / 2}$ (with $\alpha$ the shear strength) [42]. The calculated SFs change: effectively the averaged effect of the shearing is a $\sim r$ disturbance when SFs are calculated in the same direction as the velocity gradient, and additionally there is a time-dependent disturbance from the oscillation. In the sketch we also indicate why this effect was not encountered in the measurements in the central region of the cell. Due to the (on average) elliptic shape of the LSC [43], the gradient is present in the measurement area closer to the top, while falling largely outside the measurement area in the quiescent central region. It is possible to let the "sheared" SFs of Fig. 18 calculated in both directions coincide by a rotation of the coordinate frame, as is also shown in the figure. Such a rotation can unfortunately only correct for the time-independent disturbance. The time-dependent disturbance remains, contaminating the SFs.

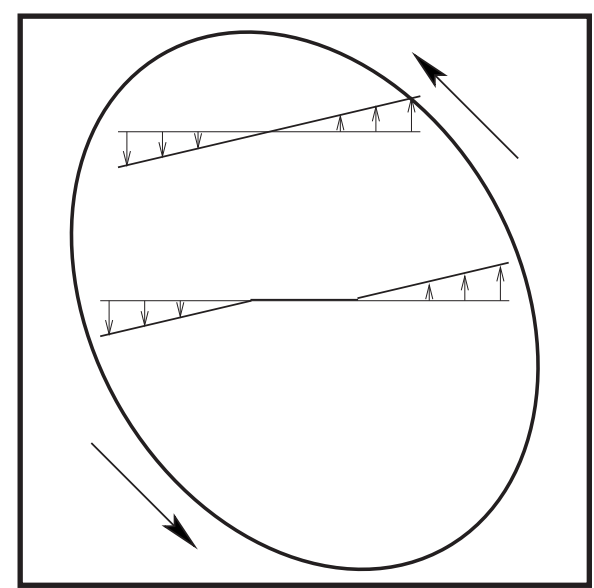

FIG. 19. Sketch of the elliptic LSC and the induced linear shear. At the off-center vertical position the gradient is present within the measurement area, while in the central region no noticeable gradient effect is found. 
Considering the shear induced by the LSC, there is an article that covers this issue [44]. It is argued there that the BO range may be indistinguishable from a shearcontaminated regime with spectral scaling $\sim k^{-7 / 3}$ and thus velocity SFs as $\sim r^{2 p / 3}$. When looking at Fig. 12 , the crosses compared to the circles, the valid scaling is clearly $\mathrm{BO}$, and not the steeper $r^{4 / 3}$ due to the shear. Indeed, the measurement area remains far from the cylinder walls, contrary to the SF of Fig. 18 where the SF indeed appears to be affected by the shear.

Indeed, a BO scaling range is found in turbulent convection, but only at rather large separations. The estimates of the Bolgiano length obtained from the numerical simulations are of the correct order. Exact compliance is not found. This is not surprising considering that $L_{B}$, formula (3), is only a dimensional estimate, and the experiment is of course different from the "idealized" numerics with perfect boundary conditions.

When comparing the current results to those of Ref. [23], it may seem that the two studies contradict each other. In that study, using a similar setup and measurement technique, only K41 and OC behavior was found. There are some important differences concerning resolution and total measurement area that explain the apparent discrepancy. In Ref. [23] the focus is on the smaller scales, with a constrained measurement area of $40 \times 40 \mathrm{~mm}^{2}$ and a superior resolution (distances between velocity vectors $0.66 \mathrm{~mm}$ ) in the center of a $H=193 \mathrm{~mm}$ cell. For the experimental settings of Ref. [23], with Ra $=1.0 \times 10^{10}$ and $\sigma=4.3$, the simulation in Sec. IV yields a local Bolgiano scale $L_{B} \approx 0.29 H$. In dimensional form, $L_{B}$ $\approx 56 \mathrm{~mm}$. Since the local Bolgiano scale is larger than the sides of the measurement area, $\mathrm{BO}$ cannot be measured and the K41 and OC scalings found in the SFs are actually the expected result. In the present study the K41 and OC scalings are not so pronounced because of a coarser resolution. But the BO scaling was found in the SFs by virtue of the larger spatial range. So this paper and Ref. [23] complement each other in that respect.

\section{CONCLUSIONS}

The structure-function scaling in turbulent convection has been investigated both numerically and experimentally.

With direct numerical simulations an analysis of the turbulent length scales in convection has been carried out. The estimates that arise from the exact relations for the dissipa- tion rates provide a good estimate for the local Kolmogorov length in the bulk. The estimate for the Bolgiano length, however, may even be off by an order of magnitude compared to the actual local values. Furthermore, this oftenemployed estimate of the Bolgiano length decreases as the Rayleigh number is increased, yet the local $L_{B}$ values in the center show an opposite correlation. Thus the interpretation of structure-function results using the global-averaged turbulent length scale estimates fails.

Velocity structure functions calculated from the simulations have shown either Bolgiano-Obukhov scaling at $\mathrm{Ra}$ $=10^{8}$ or Kolmogorov scaling for the higher Rayleigh numbers. The spatial resolution and maximal separation were inadequate for observation of both regimes at the same time. Temperature statistics were in compliance with active-scalar (Bolgiano-Obukhov) behavior.

Velocity data obtained in the experiment using SPIV were used to characterize the so-called large-scale circulation typical of confined convection. The well-known azimuthal oscillation of this circulation was described, and its oscillation period matched well with previously reported results.

The velocity data were also used for the calculation of velocity structure functions. Again, Bolgiano-Obukhov scaling was present at large separations, in line with the DNS results on the turbulent length scales. By using ESS it has been found that the relative scalings of the structure functions are also different in the two regimes.

One other suggestion that comes forward from this study is that investigations on the SF scalings could benefit from a larger-aspect-ratio cell. A larger measurement area compared to the Bolgiano scale can result in a longer scaling range. Furthermore, effects due to proximity of the sidewalls, also discussed in Ref. [23], can probably be avoided, as well as the "shearing" effects of the LSC.

\section{ACKNOWLEDGMENTS}

R.P.J.K., L.J.A.v.B., and R.A.D.A. wish to thank the Foundation for Fundamental Research on Matter [Stichting voor Fundamenteel Onderzoek der Materie (FOM)] for financial support. This work was sponsored by the National Computing Facilities Foundation (NCF) for the use of supercomputer facilities, with financial support from the Netherlands Organisation for Scientific Research (NWO). We thank D. Lohse (University of Twente) for useful remarks concerning the manuscript.
[1] A. N. Kolmogorov, Dokl. Akad. Nauk SSSR 32, 19 (1941).

[2] A. M. Obukhov, Izv. Akad. Nauk SSSR, Ser. Geogr. Geofiz. 13, 58 (1949).

[3] S. Corrsin, J. Appl. Phys. 22, 469 (1951).

[4] R. Bolgiano, J. Geophys. Res. 64, 2226 (1959).

[5] A. M. Obukhov, Dokl. Akad. Nauk SSSR 125, 1246 (1959).

[6] V. S. L'vov, Phys. Rev. Lett. 67, 687 (1991).

[7] V. Yakhot, Phys. Rev. Lett. 69, 769 (1992).

[8] A. S. Monin and A. M. Yaglom, Statistical Fluid Mechanics
(MIT Press, Cambridge, MA, 1975), Vol. 2.

[9] X.-Z. Wu, L. Kadanoff, A. Libchaber, and M. Sano, Phys. Rev. Lett. 64, 2140 (1990).

[10] R. Benzi, R. Tripiccione, F. Massaioli, S. Succi, and S. Ciliberto, Europhys. Lett. 25, 341 (1994).

[11] S. Cioni, S. Ciliberto, and J. Sommeria, Europhys. Lett. 32, 413 (1995).

[12] S. Ashkenazi and V. Steinberg, Phys. Rev. Lett. 83, 4760 (1999). 
[13] J. J. Niemela, L. Skrbek, K. R. Sreenivasan, and R. J. Donnelly, Nature (London) 404, 837 (2000).

[14] S.-Q. Zhou and K.-Q. Xia, Phys. Rev. Lett. 87, 064501 (2001).

[15] X.-D. Shang and K.-Q. Xia, Phys. Rev. E 64, 065301(R) (2001).

[16] R. Camussi and R. Verzicco, Eur. J. Mech. B/Fluids 23, 427 (2004).

[17] A. Bershadskii, K. R. Sreenivasan, and J. J. Niemela, Europhys. Lett. 69, 75 (2005)

[18] P. Tong and Y. Shen, Phys. Rev. Lett. 69, 2066 (1992).

[19] E. Calzavarini, F. Toschi, and R. Tripiccione, Phys. Rev. E 66, 016304 (2002).

[20] R. Benzi, F. Massaioli, S. Succi, and R. Tripiccione, Europhys. Lett. 28, 231 (1994).

[21] F. Chillá, S. Ciliberto, C. Innocenti, and E. Pampaloni, Nuovo Cimento D 15, 1229 (1993).

[22] T. Mashiko, Y. Tsuji, T. Mizuno, and M. Sano, Phys. Rev. E 69, 036306 (2004).

[23] C. Sun, Q. Zhou, and K.-Q. Xia, Phys. Rev. Lett. 97, 144504 (2006).

[24] B. I. Shraiman and E. D. Siggia, Phys. Rev. A 42, 3650 (1990).

[25] E. D. Siggia, Annu. Rev. Fluid Mech. 26, 137 (1994).

[26] M. Raffel, C. Willert, and J. Kompenhans, Particle Image Velocimetry (Springer, Berlin, 1998).

[27] H.-D. Xi, Q. Zhou, and K.-Q. Xia, Phys. Rev. E 73, 056312 (2006).
[28] R. Verzicco and R. Camussi, Phys. Fluids 9, 1287 (1997).

[29] R. Verzicco and R. Camussi, J. Fluid Mech. 477, 19 (2003).

[30] R. Verzicco and P. Orlandi, J. Comput. Phys. 123, 402 (1996).

[31] R. M. Kerr and J. R. Herring, J. Fluid Mech. 419, 325 (2000).

[32] R. Benzi, F. Toschi, and R. Tripiccione, J. Stat. Phys. 93, 901 (1998).

[33] S. B. Pope, Turbulent Flows (Cambridge University Press, Cambridge, England, 2000).

[34] R. Benzi, S. Ciliberto, R. Tripiccione, C. Baudet, F. Massaioli, and S. Succi, Phys. Rev. E 48, R29 (1993).

[35] L. J. A. van Bokhoven, Ph.D. thesis, Eindhoven University of Technology, 2007 (unpublished).

[36] J. J. Niemela, L. Skrbek, K. R. Sreenivasan, and R. J. Donnelly, J. Fluid Mech. 449, 169 (2001).

[37] E. Brown, A. Nikolaenko, and G. Ahlers, Phys. Rev. Lett. 95, 084503 (2005).

[38] D. Funfschilling and G. Ahlers, Phys. Rev. Lett. 92, 194502 (2004).

[39] X.-L. Qiu and P. Tong, Phys. Rev. Lett. 87, 094501 (2001).

[40] Z.-S. She and E. Leveque, Phys. Rev. Lett. 72, 336 (1994).

[41] S. Grossmann, D. Lohse, and A. Reeh, Phys. Rev. E 56, 5473 (1997).

[42] J. O. Hinze, Turbulence (McGraw-Hill, New York, 1959).

[43] C. Sun, K.-Q. Xia, and P. Tong, Phys. Rev. E 72, 026302 (2005).

[44] D. Lohse, Phys. Lett. A 196, 70 (1994). 